\title{
Development of an IoT Solution for Detergent Supervision in Industrial Washing Machines
}

\author{
Gustavo Gonçalves Coelho \\ Polytechnic Institute of Bragança \\ Campus de Santa Apolónia, \\ 5300-253 Bragança
}

\author{
Eric Henrique Moretti \\ Polytechnic Institute of Bragança \\ Campus de Santa Apolónia, \\ 5300-253 Bragança
}

\author{
João Paulo Coelho \\ Polytechnic Institute of Bragança, \\ Research Centre in Digitalization and \\ Intelligent Robotics (CeDRI) \\ Campus de Santa Apolónia, \\ 5300-253 Bragança, Portugal \\ jpcoelho@ipb.pt
}

\begin{abstract}
Automation of industrial activities aims to improve the efficiency of the productive processes while reducing costs and increasing safety. In industrial laundries, detergent management is a key factor that can lead to severe economic and environmental impacts if left uncontrolled. This paper documents the solution devised for an integrated detergent control and supervision system based on Internet-ofThings paradigms. This solution follows from a problem put forward by the laundry services of Santa Casa da Misericórdia de Bragança, located in Portugal, to the Polytechnic Institute of Bragança. In order to keep track of the detergent in a centralised dispensing system, a Wi-Fi based measurement system was developed which enables real-time monitoring of the chemicals level. In order to facilitate the physical installation of the developed hardware, a custom-made enclosure was designed and 3D printed. The acquired data is then sent to a database connected to a data processing web-based platform which is responsible for the analytics.
\end{abstract}

Keywords -Detergent Level Measurement System; Internet-of-Things; Industry 4.0; Web Development; AJAX technology;

\section{INTRODUCTION}

In the past few decades, the pressure for the companies to become increasingly competitive has grown. The demand of customized products, alongside with the decreasing product life-cycles, is making the industry to change its production paradigm [1]. These conditions, combined with the actual level in information technology, led to the emerging of a new industrial reality designated by Industry 4.0. This new stage of the manufacturing evolution aims to integrate intelligence in the production processes by adding physical objects, with embedded software and computing power [2]. These changes in the actual manufacturing model are also articulated with the emergence and development of Internet-of-Things (IoT) technologies. The technology behind IoT allows data collection from virtually any point along the production chain. This increase in information is changing the managing processes towards an effective lean manufacturing standard. The underlying concepts of Industry 4.0 are not only applicable exclusively to large industrial units. On the contrary, this new paradigm has been spreading to the entire business fabric regardless of its size. The work described throughout this document confirms this statement by providing the solution devised for an industrial laundry facility installed at the Santa Casa da Misericórdia de Bragança (SCMB), Portugal which is a private, non-profit charity institution that operates in multiple areas of social action such as health and the elderly. The actual detergent control system installed on its laundry unit is unable to cope with the operating conditions required for proper management. Due to the fact that no actual commercial product is available to solve their problem, they have challenged the Polytechnic Institute of Bragança (IPB) to devise a solution.

Knowledge transfer, from academia to the community, is a very important mission carried out by IPB leading frequently to solutions that have a very positive impact in the local businesses. For this reason, the challenge was accepted and a complete custom-made integrated detergent management system was developed. The system is composed by a set of measurement nodes (motes) installed on the detergent supply barrels which communicates, through $\mathrm{WiFi}$, with a web server. The motes are threaded to one of the two outlets of the barrels by means of a custom-made enclosure which was designed and then 3D printed. The current detergent level, at each supply unit, is measured using a low-cost ultrasonic sonar and the data is sent to a MySQL database. These data will then be used to compute several analytical figures such as the amount of detergent spent, its historical tendency, prediction regarding the expected depletion time and alerts generation, just to name a few.

In the following sections, technical details regarding each one of the developed modules, will be provided leading to the following article structure: Section II will describe in more detail the proposed problem and its requirements; Sections III and IV will be devoted to present the hardware component of the system architecture. Then, in Sections V to VII, the method used for liquid level detection will be described. Section VII deals with the hardware enclosure design solution and Section VIII describes the database structure. Section IX provides an overview on the actual functionalities provided by the web-based application platform. This article ends with Section $\mathrm{X}$ where the final remarks and future work will be presented. 


\section{PROBLEM DESCRIPTION}

The SCMB' laundry facility consists of a dozen of industrial washing machines each with a dry weight capacity of around $50 \mathrm{~kg}$. The detergent and remain chemicals needed for the washing cycles comes from a centralised dispensing room. Here, detergents, bleaches and softeners in liquid form, are stored in reservoirs with a capacity of 200 litres and driven to the washing machines via a peristaltic pump system. Fig. 1 illustrates the actual setup. In Fig. 1(a), two of the reservoirs are shown and Fig. 1(b) present the set of peristaltic pumps together with their control system.

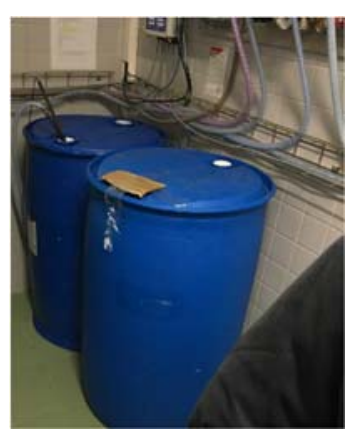

(a)

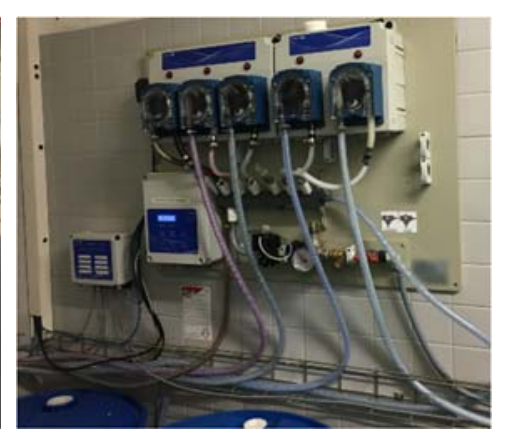

(b)
Fig. 1. The current detergent dispensing room setup: (a) High-density Polyethylene Resin (HDPE) drum barrels and (b) the set of peristaltic pumps and their associated hardware.

The state of each pump is controlled by a proprietary hardware system based on the information provided by the washing machines and a set of liquid level probes submerged in the detergent barrels. At a given time instant, the information about which type of liquid is required by a particular machine, is provided by a set of output relays accessed from the machine backside. Moreover, the level probes just provide binary information regarding the reservoir's emptiness. Notice that the individual state of each machine cannot be controlled by the actual detergent delivery system. Hence, when a given detergent product ends, the washing machine carries on its washing cycle. This will lead to a quality degradation of the cleaning process implying the repetition of the cycle in order to meet the expected washing standards. The need of those repetitions, even if not frequently occurring, has severe economic, operational and even environmental implications. On one hand, the additional electric energy spent, the machine occupation time and extra detergent doses. On the other, the delay caused by the execution of a new washing cycle and the increase in water consumption which is a sever environmental issue at the present time. Because of those problems, coupled with supplementary system limitations such as the fact that it does not allow remote access to the current state of the reservoirs, the solution to be developed should have the following set of requirements: able to measure the actual volume of detergent in the barrels; generate alerts in case this volume is not enough to allow the currently scheduled washes; allow remote access to the system state from a smartphone or other handheld device connected to the local network; be able to keep track of the detergent consumption profile and provide additional management analytic figures. Due to those requirements, a system architecture based on IoT measurement nodes was devised. The next section will deal with the technical details regarding the hardware architecture and the data storage and processing.

\section{OVERALl System ARCHITECTURE}

In the previous section, a detailed description of the problem was provided along with the set of requirements that the new solution must meet.

From a technological point-of-view, some of the concepts behind the modern Industry 4.0 paradigm could be used to ensure optimal washing results, low running cost and increasing reliability. For example, the inclusion of a smart sensing and supervision layer over the existing laundry physical setup. This added technology, supported by an effective logistics and management, will be fundamental in order to keep a sequence of events flowing, making that all necessary resources will be at the right place at the right time in the right quantity and at the right quality and cost [3].

In order to fulfil the required system conditions, the data acquisition and processing architecture presented in Fig. 2 was formulated. In this layout, a measurement node (mote) will be installed on each HDPE barrel currently under operation. Those nodes will connect, via IEEE802.11b, to a local access point. Due to the low bandwidth required by each mote, the system can scale up to 250 sensor nodes before reaching the access point limit.

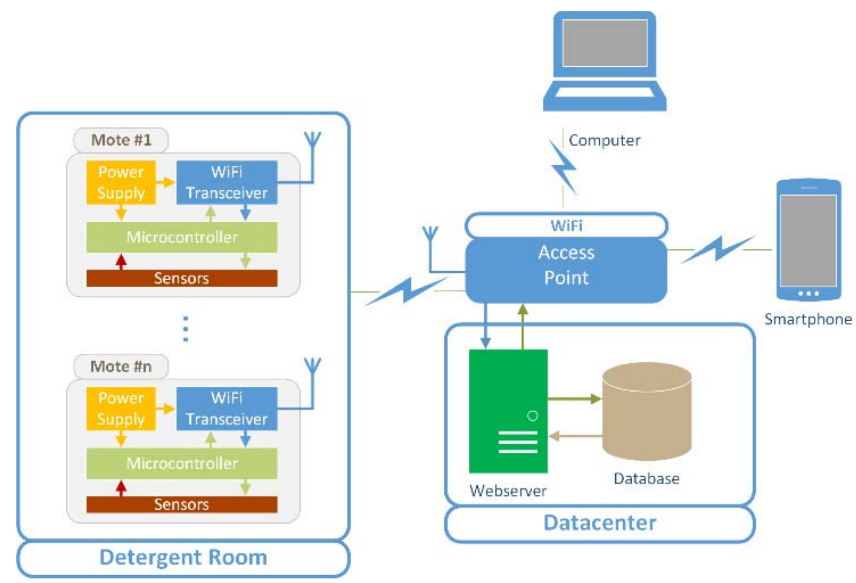

Fig. 2. Overall system architecture.

The integration of a measuring node with a detergent barrel result into cyberphysical device capable of autoawareness regarding its current status. It is able to sense changes in the detergent level and send this data to a webserver. This information will be stored into a database and will be used to compute the required analytics, generate reports and warning flags. It is also possible for smartphones, computers or other handheld devices to log into the server and access the actual system status.

The next three sections will detail the detergent level measurement system, the internal structure of the measurement nodes and the strategy adopted for their integration into the layout of the existing detergent dispensing room.

\section{MEASUREMENT NODES HARDWARE}

The purpose of this section is to describe the hardware used to build each measurement node. As can be seen from Fig. 3, each IoT measurement node relies on three modules for keeping its operation: an ultrasonic range measurement module, a 32-bit system-on-chip (SoC) device and a power supply unit. The power supply unit is composed of a lithium- 
ion (Li-Ion) battery, a battery charging and supervision module and a buck-boost DC-DC converter.

The battery used in this prototype have the standard geometry of an 18650 cell and is specified as having an open circuit voltage of $3.7 \mathrm{~V}$, a capacity of $2600 \mathrm{mAh}$ and a maximum discharge current of around $5 \mathrm{~A}$. This battery can be charged, using an off-the-shelf $5 \mathrm{~V}$ power supply, through a battery charging module. This module is built around the TC4056A chip which integrates several features such as electrical current supervision, under-voltage lockout and automatic recharge. Moreover, it also prevents the battery of over-discharging by turning it off if the voltage drops below $2.4 \mathrm{~V}$.

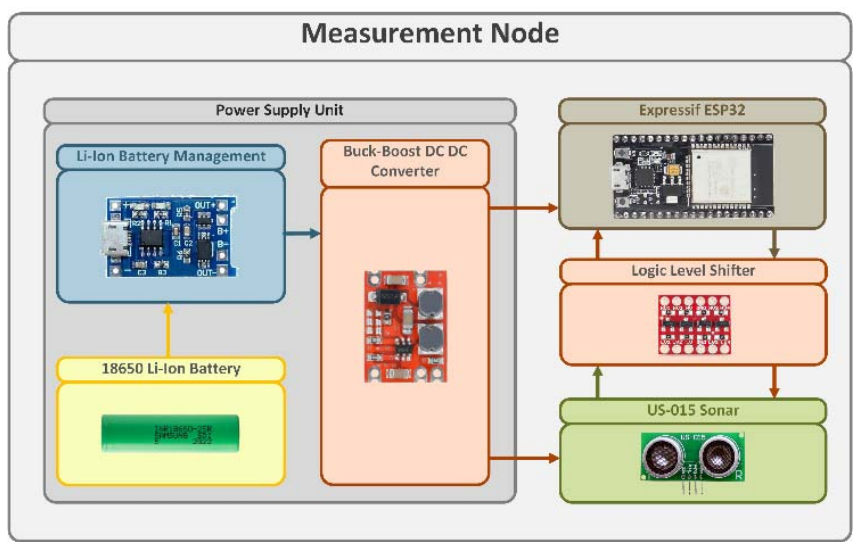

Fig. 3. Measurement nodes electronic architecture overview.

The power supply unit outputs a regulated voltage of $5 \mathrm{~V}$ which is generated from the $3.7 \mathrm{~V}$ delivered by the battery cell through a buck-boost DC-DC converter. The module selected to handle this task is able to deliver a maximum current of $600 \mathrm{~mA}$ with an output voltage ripple lower than $1 \%$.

The wireless communication, local data processing and acquisition is handled by a NodeMCU development board which is built around an ESP32 SoC. This device was developed by Espressif Systems and integrates a Tensilica Xtensa LX6 CPU with a clock frequency that can reach $240 \mathrm{MHz}$. Three main reasons have converged to make this device suitable for the current application: the integration of a WiFi transceiver, its ultra-low power consumption and price.

The information regarding the current detergent level inside the reservoir is obtained using a US-015 sonar module. The power consumption of this module is around $10 \mathrm{~mW}$ and requires a supply voltage of $5 \mathrm{~V}$. The measurement range of this sensor module extends from $2 \mathrm{~cm}$ to $4 \mathrm{~m}$. Further details regarding its working principle will be provided in the next section. Since the sonar working voltage is different from that of the NodeMCU, a logic level shifter must be used to convert the $5 \mathrm{~V}$ amplitude signal that comes from the sonar into $3.3 \mathrm{~V}$ for the microcontroller and vice-versa. The electronic schematic of the mote is presented in Fig. 4.

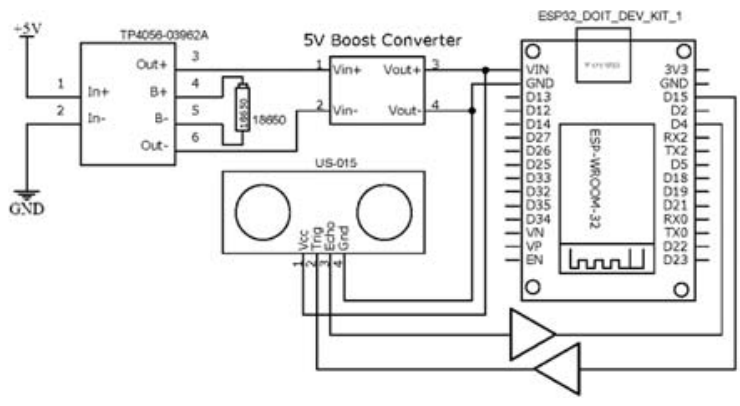

Fig. 4. Electronic circuit schematic.

As can be seen, the trigger pin of the ultrasonic sensor is connected to General Purpose Input/Output Interface (GPIO) with number D15. On the other hand, the echo pin is connected to GPIO number D4. Both connections are made through the logic level converter buffer.

Level measurement of the detergent inside the barrels is a fundamental component of the system. Be able to accurately determine the current value of the available washing products will have a severe impact on the overall system performance. For this reason, the following section will be devoted to describe the measurement method adopted.

\section{Detergent LeVel Measurement Method}

Liquid level measurement plays an important role within the devised supervisory IoT based system. For this reason, this section is devoted to describe the measurement method used and its working principle.

The measurement of the current barrel's capacity could be made using several distinct strategies. Most of them will rely on indirect measurement of the available detergent. For example, the barrels could be positioned over a weight scale then, knowing the detergent volumetric density, to infer the available capacity with its weight. However, this approach requires the proper positioning of the barrels, with more than $200 \mathrm{~kg}$ of weight, each time a replacement must be made. For this reason, a distinct measurement method based on an ultrasound sonar was considered. As can be seen from Fig. 5, on the barrel' top there are two threaded openings. One of those opening will be used for the actual system of hoses and level probes. The other will take the newly devised IoT based ultrasonic level measurement system. The system illustrated by the block diagram of Fig. 3 will be fitted into a custommade enclosure that will thread in the second opening of the barrel. Details regarding the structure of this enclosure will be present in the next section.

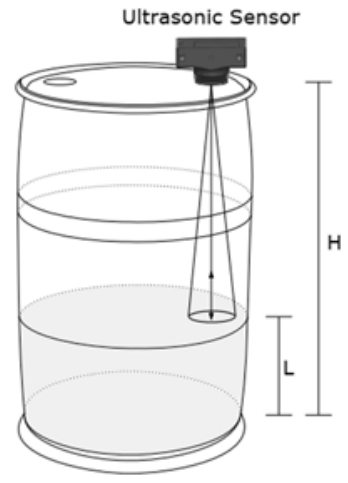

Fig. 5. Position of the ultrasonic sensor on the HDPE drum barrel. 
The main purpose of this threaded box is to place the ultrasonic sensor in a position that ensures it can send and receive ultrasonic waves without interference, principally from the plastic drum barrel's wall. Moreover, it facilitates the device changing procedure from an empty barrel to a filled one. In Fig. 5, the variable L represents the height of the liquid and $\mathrm{H}$ the height of the barrel or, to be more precise, the height of the ultrasonic sensor relatively to the reservoir. The distance between the sonar and the liquid's surface is calculated using the Time-of-Flight (ToF) method. This distance measurement method is very common within ultrasonic transducers and it is based on the time propagation of the ultrasound wave [4]. An ultrasound wave burst is emitted by the sonar and reflected back to an obstacle placed along the wave front. Knowing the speed of the sound in the medium and the time elapsed between the sent and received signal, it is possible to compute the distance between the sonar and the obstacle [5]. Hence, the distance $D$ between the sonar and the target is computed from:

$$
D=\frac{1}{2} t_{f} c_{\text {air }}
$$

where $t_{f}$ is the ToF of the ultrasonic pulse given in seconds and $c_{\text {air }}$ is the speed of sound in air given expressed in meters per second. The value of $c_{\text {air }}$ depends on the air temperature according to:

$$
c_{\text {air }}=20.05 \sqrt{T}
$$

where $T$ denotes the absolute temperature in kelvin.

As mentioned in [4], the air moisture also affects the speed of the sound wave in air. However, at a room temperature of $20{ }^{\circ} \mathrm{C}$, its impact is just around $0.15 \%$. For this reason, measurement compensation due to air moisture was disregarded during the present work. Another consideration which must be taken into account is the wave's propagation path [6]. As can be seen for Fig. 6, due to the sonar's geometry the distance between the sensor and the obstacle requires to find the height of an isosceles triangle.

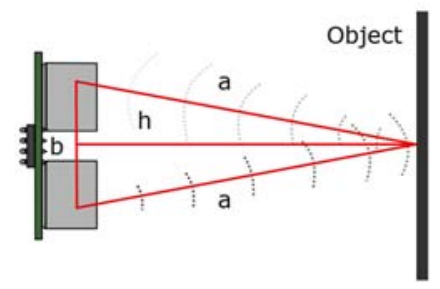

Fig. 6. Geometric representation of the sound wave path emitted and reflected by an obstacle (adapted from [6]).

For the present prototype, the US-015 sonar was considered. This device is manufactured by Synacorp Technologies and, regarding the geometry of Fig. 6, the distance $b$ is equal to $2.3 \mathrm{~cm}$ which leads to the following distance measurement equation

$$
h=\sqrt{a^{2}-1.32}
$$

with both $h$ and $a$ measured in centimetres. Considering the barrel as a regular cylinder, the liquid volume can be estimated by:

$$
V=\pi \frac{d^{2}(H-h)}{4000}
$$

where $V$ is the actual liquid volume measured in litres, $d$ is the barrel diameter expressed in centimetres and $H$ the barrel's height also in centimetres. Notice that, since the barrel is not a perfect cylinder, $d V / d h$ is not constant. However, the average volume error is lower than $5 \%$ if (4) is employed to compute the instantaneous volume of remaining liquid.

The following section will present the experimental data obtained with this sensor and characterize the metrological behaviour of the sonar.

\section{SONAR MEASUREMENT BEHAVIOUR}

In order to characterize the sonar accuracy and repeatability, the device was used to acquire 1000 samples over a target object placed at three distinct distances: $10 \mathrm{~cm}$, $50 \mathrm{~cm}$ and $90 \mathrm{~cm}$. It is worth to notice that all the tests were performed in a controlled environment where the temperature was kept constant. The results collected from the samples are presented in Table 1. The first column represents the samples mean, the second the standard deviation, the third the systematic error $\left(S_{E}\right)$ and the fourth the relative error $(\varepsilon)$.

\begin{tabular}{|c|c|c|c|c|}
\hline Distance (cm) & $\overline{\boldsymbol{x}} \mathbf{( \mathbf { c m } )}$ & $\boldsymbol{\sigma}_{\boldsymbol{s}} \mathbf{( c m )}$ & $\boldsymbol{S}_{\boldsymbol{E}} \mathbf{( c m )}$ & $\boldsymbol{\varepsilon} \mathbf{( \% )}$ \\
\hline $\mathbf{1 0}$ & 11.01 & 0.03 & 1.01 & 10.08 \\
\hline $\mathbf{5 0}$ & 51.63 & 0.06 & 1.63 & 3.27 \\
\hline $\mathbf{9 0}$ & 91.73 & 0.07 & 1.73 & 1.92 \\
\hline
\end{tabular}

Table 1. Summary of the result values from the 1000 samples collected.

As can be noticed, for longer distances, the samples have higher standard deviation values. Moreover, shorted distances exhibit larger relative errors. In order to characterize the stochastic measurement component, 15 samples were randomly selected from the available samples. Assuming a $95 \%$ confidence interval, the measurement samples can be decomposed by [11]:

$$
M R=\bar{x}-S_{E} \pm \frac{R e p}{\sqrt{n}}
$$

where $n$ is the number of samples and Rep is the repeatability which is defined as,

$$
R e p= \pm t x \sigma_{s}
$$

in which $t$ is the Student's t distribution. Therefore, for $n=$ 15 samples, the Student's-t table delivers a value of $t=2.14$ for 14 degree of freedom and 95\% confidence interval. Thereby, it is possible to estimate the system repeatability and define the stochastic component as demonstrated in Table 2.

\begin{tabular}{|c|c|c|c|c|c|}
\hline Distance (cm) & $\overline{\boldsymbol{x}} \mathbf{( c m )}$ & $\boldsymbol{\sigma}_{\boldsymbol{S}}(\mathbf{c m})$ & $\boldsymbol{S}_{\boldsymbol{E}}(\mathbf{c m})$ & $\boldsymbol{R e}(\mathbf{c m})$ & $\boldsymbol{M R} \mathbf{( c m )}$ \\
\hline $\mathbf{1 0}$ & 11.02 & 0.02 & 1.02 & 0.04 & $10 \pm 0.01$ \\
\hline $\mathbf{5 0}$ & 51.65 & 0.07 & 1.65 & 0.1 & $50 \pm 0.03$ \\
\hline $\mathbf{9 0}$ & 91.71 & 0.06 & 1.71 & 0.1 & $90 \pm 0.03$ \\
\hline
\end{tabular}

Table 2. Result using a confidence interval of $95 \%$.

From the results it can be noticed that, for distances over $50 \mathrm{~cm}$, there is a $95 \%$ of confidence that the measurement values are within $\pm 0.03 \mathrm{~cm}$. Already for the distance $10 \mathrm{~cm}$, the range is shorter, presented a range of values within \pm 0.01 $\mathrm{cm}$. These degree of accuracy and repeatability is way higher than required in the actual detergent supervision application which justifies the choice of this sonar as the backbone of our measurement system. It is worth to notice that, using this average filtering technique, the system requires a total time of 2.6 seconds to take the readings from the detergent's liquid surface. However, due to the system time constants, this 
processing time is within the required measurement system bandwidth.

It is important to highlight that, one of the design constraints, is to produce a non-invasive measurement system, physically robust and able to be easily switched from one empty barrel to a full one. Those conditions had led to the development of a custom hardware enclosure which will be detailed during the next section.

\section{ENCLOSURE DESIGN}

This section deals with the solution devised for the IoT hardware enclosure. For prototype and proof-of-concept, the case was designed using SolidWorks ${ }^{\circledR}$ software and them 3D printed using PLA filament. The case can be viewed and having two parts. One is a threaded cap which will hold the sonar sensor and the other is a regular box where all the electronic modules will be fitted. Starting with the screw thread, by manually performing the measurements of diameter, pitch and thread shape using a digital calliper, the 3D body illustrated in Fig 7 was designed.

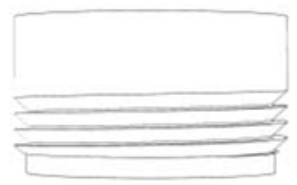

(a) Front view.

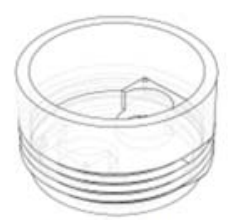

(b) Isometric view.
Fig. 7. Overview of the threaded cap designed where, in (b), it is possible the view the sonar accommodation place.

Then, using a Fusion3 F400 3D printer, the threaded cap was printed using PLA filament. The final result can be seen on Fig. 8.

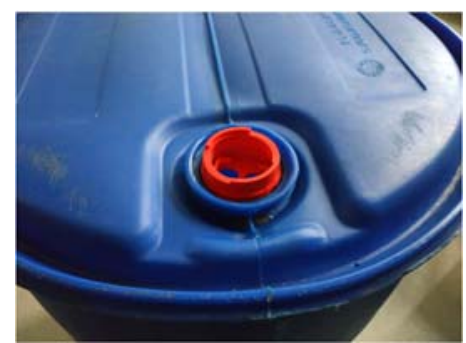

Fig. 8. Printed 3D threaded cap being tested on a barrel.

After fine tuning the dimensions of the threaded cap, the complete hardware enclosure case was designed. The image from Fig. 9 present two views of the final solution. As can be seen, two access openings are available: one for the NodeMCU and the other for the battery module charger. In the middle, a switch button is placed for turning the device on and off. In the side view, the openings for the onboard LEDs are made visible. Those LED will be responsible for showing the current system status such as if the device is powered up and if a liquid level warning is currently active.

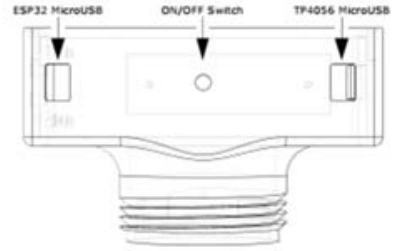

(a) Front view.

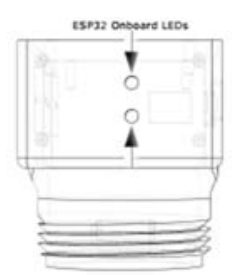

(b) Side view.
Fig. 9. Preview of the 3D device model.

The final version of the device, showing the position of the electronic modules, is presented in Fig. 10 and the final 3D printed model, with the hardware already embedded in the enclosure, can be viewed in Fig. 11.

After describing the hardware related to this project, the next two section will be intended to describe the information processing and data storage platform.

\section{DATABase DeVElopment}

This section will describe the structure of the database and the APIs responsible for allowing the communication between the client and the data server. It is worth to notice that the source codes developed and described in this section can be downloaded at [7].

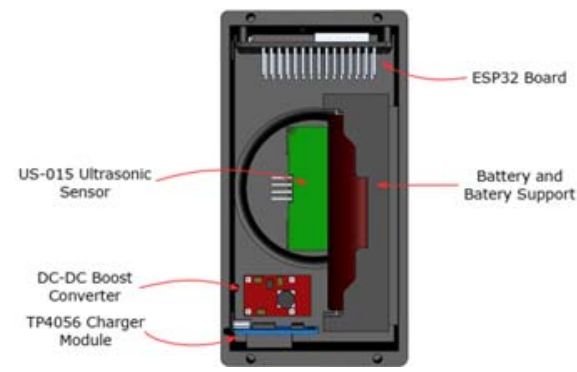

Fig. 10. Layout of the electronic components.

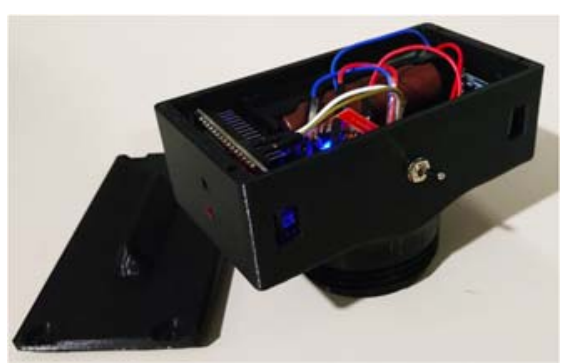

Fig. 11. Final version of the 3D printed model.

A three-tier server architecture, as presented in Fig. 12, was used in this project. The Tier 0 represents the client side, which can be any machine with user capabilities and local processing. Tier 1 is responsible for enabling the connection between the client and the server through HTTP protocols programmed in PHP. Lastly, the server side is represented by the Tier 2, which contains both the hardware and software responsible for the data storage [8]. 


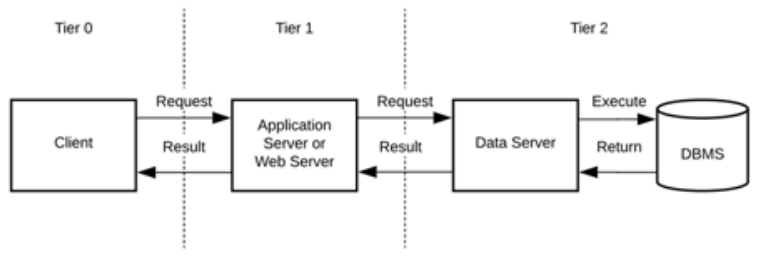

Fig. 12. Logical three-tier client/server architecture (adapted from [8]).

After defining the database logical structure, the server architecture, illustrated in Fig. 13, was devised. As can been seen, the APIs allow the users to access the database content, dynamically and interactively, through the HTML web pages.

The laundry managements can access the database content by logging into a website whose structure is presented in Fig 14. The communications are done by using HTTP protocols, mostly through the GET and POST methods and the server's response is in JSON format [9]. Details regarding this webpage will be provided in the next section.

\section{Website Structure}

In this section, some of the services provided by website will be described. In this website, all the graphs were implemented using the Google Chart API, which is a powerful free service. In Início (Start), it is possible to check the actual reservoir capacity and current status. It also presents the predictions regarding the time estimated for full depletion of a particular detergent. Fig. 15 illustrates this functionality. The predictions are made using the secant numerical method [10] and are provided by a custom-made PHP API.

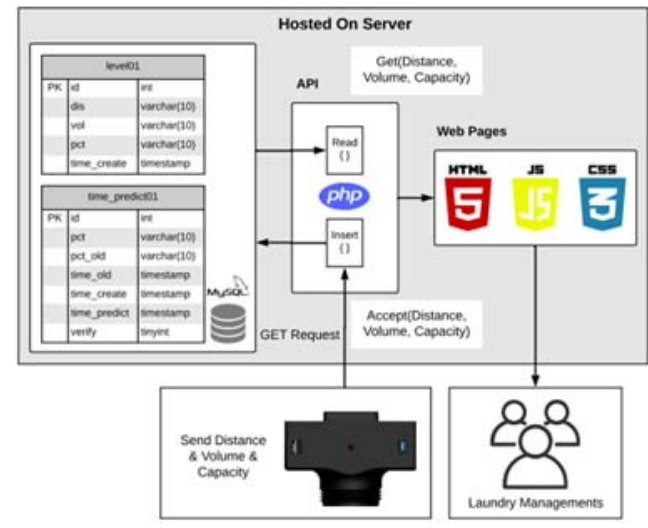

Fig. 13. Web database architecture overview.

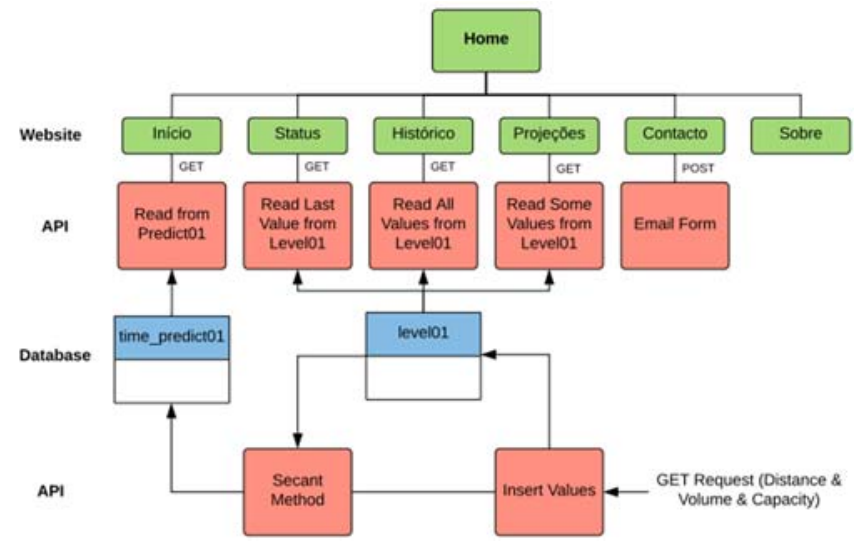

Fig. 14. Website structure diagram.

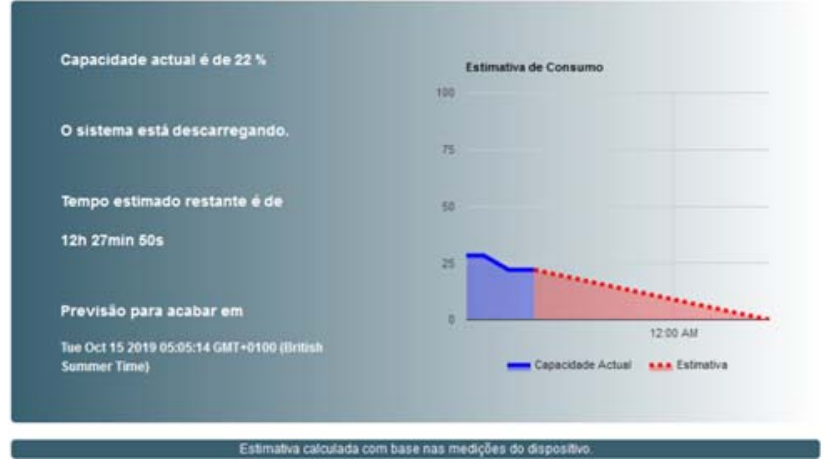

Fig. 15. Information of the expected time to full depletion of the reservoir.

The information is update by using AJAX technique, which asynchronously requests the server to refresh a portion of the browser display, instead of loading the entire web page [10]. This feature makes the webpage information to be dynamically updated. Many more functionalities and data analytics are available. For example, in Status option, the customer has access to the last measurement values such as the raw sensor distance, the estimated barrel volume, the remain capacity and current time-stamp. On the other hand, Histórico (Historical) allows the user to navigate through the whole database content, enabling sort and search. The lefthand side image of Fig. 16 presents the Status window and the right-hand side the Historical data.

\section{CONCLUSIONS}

This paper describes the technical solutions to solve a realworld problem regarding the management of detergents for an industrial laundry room. An IoT approach was taken leading to the development of a hardware measurement node and a data analytics and storage web-based application. This application strongly relies on several PHP APIs which demonstrates to be efficient, capable of efficiently read large volumes of data. Moreover, the use of AJAX enables a smooth match with the IoT hardware enabling the web application to dynamically respond to data changes transmitted by the motes. The devised solution does not require any modification on the existent layout at the laundry facility and it behaves like an easily scalable plug-and-play solution.

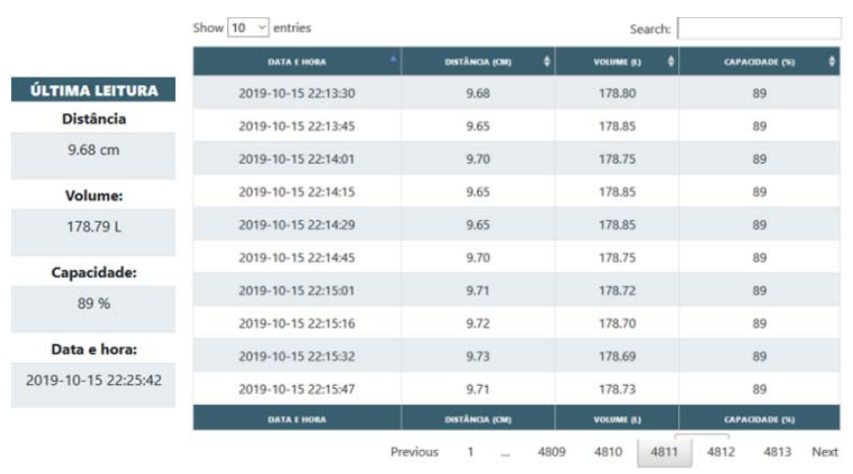

Fig. 16. At left-hand side, the Status layout and at righthand side the Historical data.

Future system improvements will be made toward encryption and data security. For example, resorting to API protection such as REST or SOAP. Moreover, more robust materials must be considered to increase the enclosure mechanical robustness. 


\section{REFERENCES}

[1] M. Brettel, N. Friederichsen, M. Keller, and M. Rosenberg, "How virtualization, decentralization and network building change the manufacturing landscape: An industry 4.0 perspective", International Journal of Mechanical, vol. 7, no. 1, Nov. 2014.

[2] F. Almada-Lobo, "The industry 4.0 revolution and the future of manufacturing execution systems", Journal of Innovation Management, vol. 3, pp. 16-21. Jan. 2016.

[3] A. E. Branch, "Global supply chain management and international logistics”, ISBN: 0415398452, Feb 2009

[4] A. Carullo and M. Parvis, "An ultrasonic sensor for distance measurement in automotive applications", Sensors Journal, IEEE, vol. 1, pp. 143-147, Sep. 2001. doi: 10.1109/JSEN.2001.936931.

[5] K. N. D. Grassi, R. C. S. Freire, and J. M. M. Villanueva, "Evaluation of wavelet analysis performance in multiphase level measurement using ultrasonic sensors", IMT Conf., May 2014, pp. 756-760.
[6] V. Zhmud, N. Kondratiev, K. Kuznetsov, V. Trubin, and L. Dimitrov, "Application of ultrasonic sensor for measuring distances in robotics", Journal of Physics, vol. 1015, p. 032 189, May 2018.

[7] G. G. Coelho, Developed Codes for an IoT solution for detergent supervision in industrial washing machines, version v1.0.0, Oct. 2019.

[8] S. B. N. Ramez Elmasri, Fundamentals of database systems, 7th ed. Pearson, 2015, ISBN: 978-0133970777.

[9] T. Felke-Morris, Web development and design foundations with html5, 9th, ser.Web development and Design Foundations with HTML5. Pearson, 2019, ISBN:870134801148.

[10] K. A. Eldrandaly, M. Abdel-Basset, and L. A. Shawky, "Internet of spatial things: A new reference model with insight analysis", IEEE Access, vol. 7, pp. 19 653-19 669, 2019, ISSN: 2169-3536.

[11] J. Neto, Metrologia e controle dimensional. conceitos, normas e aplicação.2012, ISBN: 978-85-352-5579-9. 